\title{
The Existence of Heteroclinic Travelling Waves in the Discrete Sine-Gordon Equation with Nonlinear Interaction on a 2D-Lattice
}

\begin{abstract}
S. Bak
The article deals with the discrete sine-Gordon equation that describes an infinite system of nonlinearly coupled nonlinear oscillators on a 2D-lattice with the external potential $V(r)=K(1-\cos r)$. The main result concerns the existence of heteroclinic travelling waves solutions. Sufficient conditions for the existence of these solutions are obtained by using the critical points method and concentration-compactness principle.
\end{abstract}

Key words: discrete sine-Gordon equation, nonlinear oscillators, 2D-lattice, heteroclinic travelling waves, critical points, concentration-compactness principle.

Mathematical Subject Classification 2010: 34G20, 37K60, 58E50.

\section{Introduction}

In the paper, we study the discrete sine-Gordon equation that describes the dynamics of an infinite system of nonlinearly coupled nonlinear oscillators on a two-dimensional lattice. Let $q_{n, m}$ be a generalized coordinate of the $(n, m)$-th oscillator at the time $t$. It is assumed that each oscillator interacts nonlinearly with its four nearest neighbors. The equation of motion of the system considered is of the form

$$
\begin{aligned}
\ddot{q}_{n, m}= & V^{\prime}\left(q_{n+1, m}-q_{n, m}\right)-V^{\prime}\left(q_{n, m}-q_{n-1, m}\right)+V^{\prime}\left(q_{n, m+1}-q_{n, m}\right) \\
& -V^{\prime}\left(q_{n, m}-q_{n, m-1}\right)-K \sin \left(q_{n, m}\right), \quad(n, m) \in \mathbb{Z}^{2},
\end{aligned}
$$

where $K>0$. Equations (1) form an infinite system of ordinary differential equations.

System (1) can be considered as a 2D version of the Frenkel-Kontorova model (see, e.g., [11]). Notice that this system represents a wide class of systems called lattice dynamical systems extensively studied in last decades. In this area of research, a great attention is paid to an important specific class of solutions called travelling waves solutions. A comprehensive presentation of the results on travelling waves for 1D Fermi-Pasta-Ulam lattices is given in [19]. The existence 
of periodic travelling waves in the Fermi-Pasta-Ulam system on a 2D-lattice is studied in [4]. On the other hand, some results on the chains of oscillators are also known in the literature. In particular, in [14] they are obtained by means of bifurcation theory, while in [1] and [2] the existence of periodic and solitary travelling waves is studied by means of the critical point theory. In papers $[3,10,12,13]$, travelling waves for infinite systems of linearly coupled oscillators on a 2D-lattice are studied. Paper [18] is devoted to periodic and homoclinic travelling waves for the infinite one-dimensional chain of nonlinearly coupled nonlinear particles. In [6], a result on the existence of subsonic periodic travelling waves for the system of nonlinearly coupled nonlinear oscillators on a 2D-lattice is obtained, and in [7], supersonic periodic travelling waves for these systems are studied. Paper [15] contains a result on the existence of heteroclinic travelling waves for the discrete sine-Gordon equation with linear interaction. In [16], periodic, homoclinic and heteroclinic travelling waves for such systems with nonlinear interaction are studied. In paper [5], a result on the existence of periodic travelling waves for the discrete sine-Gordon equation with nonlinear interaction on a 2D-lattice is obtained. [8] is devoted to the existence of heteroclinic travelling waves for the discrete sine-Gordon equation with linear interaction on a 2D-lattice.

\section{The problem statement}

A travelling wave solution of equation (1) is a function of the form

$$
q_{n, m}(t)=u(n \cos \varphi+m \sin \varphi-c t),
$$

where the profile function $u(s)$ of the wave, or simply profile, satisfies the equation

$$
\begin{aligned}
c^{2} u^{\prime \prime}(s)= & V^{\prime}(u(s+\cos \varphi)-u(s))-V^{\prime}(u(s)-u(s-\cos \varphi)) \\
& +V^{\prime}(u(s+\sin \varphi)-u(s))-V^{\prime}(u(s)-u(s-\sin \varphi))-K \sin (u(s)) .
\end{aligned}
$$

The constant $c \neq 0$ is called the speed of the wave. If $c>0$, then the wave moves to the right, otherwise to the left.

An important role is played by the quantity $c_{1}$ defined by the equation

$$
c_{1}^{2}:=2 \sup _{|r|<6 \pi}\left|\frac{V(r)}{r^{2}}\right| .
$$

We consider the case of heteroclinic travelling waves. The profile function of this wave satisfies the conditions:

$$
\lim _{s \rightarrow-\infty} u(s)=-\pi \quad \text { and } \quad \lim _{s \rightarrow+\infty} u(s)=\pi .
$$

In what follows, a solution of equation (2) is understood as a function $u(s)$ from the space $C^{2}(\mathbb{R})$ satisfying equation (2) for all $s \in \mathbb{R}$. 


\section{Variational setting}

To equation (2), we associate the functional

$$
\begin{aligned}
J(u):=\int_{-\infty}^{+\infty} & {\left[\frac{c^{2}}{2}\left(u^{\prime}(s)\right)^{2}-V(u(s+\cos \varphi)-u(s))\right.} \\
& -V(u(s+\sin \varphi)-u(s))+K(1+\cos (u(s)))] d s,
\end{aligned}
$$

defined on the Hilbert space

$$
E:=\left\{u \in H_{\mathrm{loc}}^{1}(\mathbb{R}): u^{\prime} \in L^{2}(\mathbb{R})\right\}
$$

with the scalar product

$$
(u, v)_{E}=u(0) v(0)+\int_{-\infty}^{+\infty} u^{\prime}(s) v^{\prime}(s) d s .
$$

It is not so difficult to verify that the critical points of the functional $J$ are the solutions of equation (2).

Now we introduce the following notation:

$$
\begin{aligned}
\mathcal{M}_{-\pi, \pi} & =\{u \in E: u(-\infty)=-\pi, u(+\infty)=\pi\} \\
A u(s) & :=u(s+\cos \varphi)-u(s), \\
B u(s) & :=u(s+\sin \varphi)-u(s) .
\end{aligned}
$$

According to Lemma 3.1 from [10],

$$
\begin{aligned}
\|A u(s)\|_{L^{2}(\mathbb{R})} & \leq|\cos \varphi| \cdot\left\|u^{\prime}(s)\right\|_{L^{2}(\mathbb{R})}, & & u \in E, \\
\|B u(s)\|_{L^{2}(\mathbb{R})} & \leq|\sin \varphi| \cdot\left\|u^{\prime}(s)\right\|_{L^{2}(\mathbb{R})}, & & u \in E .
\end{aligned}
$$

Then the functional $J$ can be expressed in the form

$$
J(u):=\int_{-\infty}^{+\infty}\left[\frac{c^{2}}{2}\left(u^{\prime}(s)\right)^{2}-V(A u(s))-V(B u(s))+K(1+\cos (u(s)))\right] d s .
$$

Throughout the paper we will assume that the interaction potential $V(r)$ satisfies the following conditions:

(i) $V(r) \in C^{1}(\mathbb{R}), V(0)=0$ and $V(r) \geq 0$ for all $r \in \mathbb{R}$;

(ii) $\lim _{r \rightarrow \pm \infty} V(r)=+\infty$;

(iii) there exists finite $\lim _{r \rightarrow 0}\left|\frac{V(r)}{r^{2}}\right|$;

(iv) the wave speed $c$ satisfies $c^{2}>c_{1}^{2}$.

The following lemma can be obtained by a straightforward calculation (see [15] for details). 
Lemma 3.1. Let $v_{0}: \mathbb{R} \rightarrow[-\pi, \pi]$ be a monotone function in $C^{\infty}(\mathbb{R})$ such that $v_{0}(s)=-\pi$ for $s<-1$ and $v_{0}(s)=\pi$ for $s>1$. Define the functional $\Psi$ : $H^{1}(\mathbb{R}) \rightarrow \mathbb{R}$ by

$$
\Psi(v):=J\left(v_{0}+v\right)
$$

and suppose that assumptions (i)-(iv) are satisfied. Then the following holds:

(i $\left.i_{1}\right) \Psi(v)<+\infty$ for all $v \in H^{1}(\mathbb{R})$ (equivalently, $J(u)<+\infty$ for all $u$ of the form $u=v_{0}+v$ for some $\left.v \in H^{1}(\mathbb{R})\right)$;

(ii $\left.{ }_{1}\right) J(u)=+\infty$ for all $u \in \mathcal{M}_{-\pi, \pi}$ which are not of the form $u=v_{0}+v$ for some $v \in H^{1}(\mathbb{R})$. In particular, a minimizer $u$ of $J$ on $\mathcal{M}_{-\pi, \pi}$ can be expressed as $u=v_{0}+v$ for some $v \in H^{1}(\mathbb{R})$;

(iii 1$) \Psi \in C^{1}$ on $H^{1}(\mathbb{R})$;

(ivi) let $v \in H^{1}(\mathbb{R})$ be a critical point of $\Psi$ and set $u:=v_{0}+v$. Then $u, v \in$ $C^{2}(\mathbb{R})$, and $u$ is a solution of (2) with boundary conditions (3).

Let $F$ be a non-negative function in $C^{\infty}(\mathbb{R})$ such that

$$
\begin{cases}F(r)=0, & \text { if }|r| \leq \frac{5 \pi}{2} \\ F(r) \geq 4\left|\int_{0}^{2 r}\right| V^{\prime}(x)|d x| \text { and } F(r) \geq 2 K, & \text { if }|r| \geq 3 \pi \\ \frac{1}{2} \leq 1+\cos r+\frac{1}{2 K} F(r), & \text { if }|r| \in\left(\frac{5}{2} \pi, 3 \pi\right) .\end{cases}
$$

Now we define the modified functional $\tilde{J}: E \rightarrow \mathbb{R} \cup\{\infty\}$ by

$$
\begin{aligned}
\tilde{J}(u):=\int_{-\infty}^{+\infty}\left[\frac{c^{2}}{2}\left(u^{\prime}(s)\right)^{2}\right. & -V(A u(s))-V(B u(s)) \\
& +K(1+\cos (u(s)))+F(u(s))] d s .
\end{aligned}
$$

Remark 3.2. Obviously, $\tilde{J}(u)=J(u)$ for all $u \in E$ with norm

$$
\|u\|_{L^{\infty}(\mathbb{R})} \leq \frac{5}{2} \pi
$$

Now we denote the modified potential of interaction by

$$
\tilde{V}(r)=\left|\int_{0}^{r}\right| V^{\prime}(x)|d x| .
$$

Then from (6) for all $|r| \geq 3 \pi$, we have

$$
V(2 r) \leq \tilde{V}(2 r) \leq \frac{1}{4} F(r)
$$

Hence, by (ii), $F(r) \rightarrow+\infty$ for $r \rightarrow \pm \infty$.

The lemma below can be found in [16, Lemma 2.5]. 
Lemma 3.3. Let $W \in C^{1}(\mathbb{R})$ be such that $W( \pm \pi)=0$ and $W(\xi)>0$ for $|\xi|<\pi$, and let

$$
I(u):=\int_{-\infty}^{+\infty}\left[\left(u^{\prime}(s)\right)^{2}+W(u(s))\right] d s .
$$

Then the minimum of $I$ on $\mathcal{M}_{-\pi, \pi}$ is attained and

$$
\min _{u \in \mathcal{M}} I(u)=2 \int_{-\pi}^{\pi} \sqrt{W(\xi)} d \xi=: \vartheta
$$

Moreover, with the same $\vartheta$,

$$
\inf _{T>0} \inf _{u \in H^{1}(-T, T)}\left\{\int_{-T}^{T}\left[\left(u^{\prime}(s)\right)^{2}+W(u(s))\right] d s: u(-T)=-\pi, u(T)=\pi\right\}=\vartheta .
$$

Lemma 3.4. Assume conditions (i)-(iv) hold. Then for all $u \in E$,

$$
\tilde{J}(u) \geq \int_{-\infty}^{+\infty}\left[\frac{c^{2}-c_{1}^{2}}{2}\left(u^{\prime}(s)\right)^{2}+K\left(1+\cos (u(s))+\frac{1}{2} F(u(s))\right] d s,\right.
$$

and the functional $\tilde{J}$ is bounded from below on $\mathcal{M}_{-\pi, \pi}$. Moreover,

$$
8 \sqrt{\left(c^{2}-c_{1}^{2}\right) K}<\inf _{u \in \mathcal{M}} \tilde{J}(u)<8 c \sqrt{K} .
$$

Proof. Since

$$
\begin{aligned}
& |A u(s)| \leq|u(s+\cos \varphi)|+|u(s)| \leq 2 \max \{|u(s+\cos \varphi)|,|u(s)|\}, \\
& |B u(s)| \leq|u(s+\sin \varphi)|+|u(s)| \leq 2 \max \{|u(s+\sin \varphi)|,|u(s)|\},
\end{aligned}
$$

then for every $k>0$,

$$
\begin{aligned}
\{s \in \mathbb{R}:|A u(s)|>k\} & \subseteq\left\{s \in \mathbb{R}: \max \{|u(s+\cos \varphi)|,|u(s)|\}>\frac{k}{2}\right\} \\
& \subseteq\left\{s \in \mathbb{R}:|u(s+\cos \varphi)|>\frac{k}{2}\right\} \cup\left\{s \in \mathbb{R}:|u(s)|>\frac{k}{2}\right\}, \\
\{s \in \mathbb{R}:|B u(s)|>k\} & \subseteq\left\{s \in \mathbb{R}: \max \{|u(s+\sin \varphi)|,|u(s)|\}>\frac{k}{2}\right\} \\
& \subseteq\left\{s \in \mathbb{R}:|u(s+\sin \varphi)|>\frac{k}{2}\right\} \cup\left\{s \in \mathbb{R}:|u(s)|>\frac{k}{2}\right\} .
\end{aligned}
$$

Making use of $(8)$ and the monotonicity of the potential $\tilde{V}$ on $(-\infty, 0)$ and on $(0,+\infty)$, we have

$$
\begin{aligned}
& \int_{\{s \in \mathbb{R}:|A u(s)|>6 \pi\}} V(A u(s)) d s \leq \int_{\{s \in \mathbb{R}:|A u(s)|>6 \pi\}} \tilde{V}(A u(s)) d s \\
& \leq \int_{\{s \in \mathbb{R}:|A u(s)|>6 \pi\}} \tilde{V}(2 \max \{|u(s+\cos \varphi)|,|u(s)|\}) d s
\end{aligned}
$$




$$
\begin{aligned}
& \leq \int_{\{s \in \mathbb{R}: \max \{|u(s+\cos \varphi)|,|u(s)|\}>3 \pi\}} \frac{1}{4} F(\max \{|u(s+\cos \varphi)|,|u(s)|\}) d s \\
& \leq 2 \int_{\{s \in \mathbb{R}:|u(s)|>3 \pi\}} \frac{1}{4} F(u(s)) d s \leq \frac{1}{2} \int_{-\infty}^{+\infty} F(u(s)) d s .
\end{aligned}
$$

Similarly,

$$
\int_{\{s \in \mathbb{R}:|B u(s)|>6 \pi\}} V(B u(s)) d s \leq \frac{1}{2} \int_{-\infty}^{+\infty} F(u(s)) d s .
$$

By the definition of $c_{1}$, we obtain

$$
\begin{aligned}
\int_{\{s \in \mathbb{R}:|A u(s)| \leq 6 \pi\}} V(A u(s)) d s & \leq \int_{\{s \in \mathbb{R}:|A u(s)| \leq 6 \pi\}} \frac{c_{1}^{2}}{2}(A u(s))^{2} d s \\
& \leq \int_{-\infty}^{+\infty} \frac{c_{1}^{2}}{2}(A u(s))^{2} d s \\
\int_{\{s \in \mathbb{R}:|B u(s)| \leq 6 \pi\}} V(B u(s)) d s & \leq \int_{\{s \in \mathbb{R}:|B u(s)| \leq 6 \pi\}} \frac{c_{1}^{2}}{2}(B u(s))^{2} d s \\
& \leq \int_{-\infty}^{+\infty} \frac{c_{1}^{2}}{2}(B u(s))^{2} d s .
\end{aligned}
$$

Then it follows from (11) and (12) that

$$
\begin{aligned}
\tilde{J}(u) \geq & \int_{-\infty}^{+\infty}\left[\frac{c^{2}}{2}\left(u^{\prime}(s)\right)^{2}-\frac{c_{1}^{2}}{2}(A u(s))^{2}-\frac{c_{1}^{2}}{2}(B u(s))^{2}\right. \\
& +K(1+\cos (u(s)))+F(u(s))] d s \\
& -\int_{\{s \in \mathbb{R}:|A u(s)|>6 \pi\}} V(A u(s)) d s-\int_{\{s \in \mathbb{R}:|B u(s)|>6 \pi\}} V(B u(s)) d s \\
\geq & \int_{-\infty}^{+\infty}\left[\frac{c^{2}-c_{1}^{2}}{2}\left(u^{\prime}(s)\right)^{2}+K(1+\cos (u(s)))+\frac{1}{2} F(u(s))\right] d s
\end{aligned}
$$

for all $u \in E$, and (9) holds true.

Applying Lemma 3.3 to the functional

$$
I_{1}(u)=\frac{c^{2}-c_{1}^{2}}{2} \int_{-\infty}^{+\infty}\left[\left(u^{\prime}(s)\right)^{2}+W_{1}(u(s))\right] d s,
$$

where

$$
W_{1}(x):=\frac{2 K}{c^{2}-c_{1}^{2}}\left[1+\cos x+\frac{1}{2 K} F(x)\right],
$$

and making use of (9), we obtain

$$
\begin{aligned}
\inf _{u \in \mathcal{M}_{-\pi, \pi}} \tilde{J}(u) & \geq\left(c^{2}-c_{1}^{2}\right)\left|\int_{-\pi}^{\pi} \sqrt{W_{1}(x)} d x\right| \\
& =\sqrt{2\left(c^{2}-c_{1}^{2}\right) K}\left|\int_{-\pi}^{\pi} \sqrt{1+\cos x+0} d x\right|=8 \sqrt{\left(c^{2}-c_{1}^{2}\right) K} .
\end{aligned}
$$


Furthermore, since $V \geq 0$, we have

$$
\tilde{J}(u) \leq \frac{c^{2}}{2} \int_{-\infty}^{+\infty}\left[\left(u^{\prime}(s)\right)^{2}+\frac{2}{c^{2}}\left(K(1+\cos (u(s)))+\frac{3}{2} F(u(s))\right)\right] d s .
$$

Now, we apply Lemma 3.3 to the functional

$$
I_{2}(u)=\frac{c^{2}-c_{1}^{2}}{2} \int_{-\infty}^{+\infty}\left[\left(u^{\prime}(s)\right)^{2}+W_{2}(u(s))\right] d s,
$$

where

$$
W_{2}(x):=\frac{2 K}{c^{2}}\left[1+\cos x+\frac{3}{2 K} F(x)\right] .
$$

As a consequence, we obtain

$$
\inf _{u \in \mathcal{M}_{-\pi, \pi}} \tilde{J}(u) \leq c^{2}\left|\int_{-\pi}^{\pi} \sqrt{W_{2}(x)} d x\right|<8 c \sqrt{K}
$$

from which inequalities (10) follow.

The following lemma can be proved in the same way as Lemma 2.7 from [16].

Lemma 3.5. Assume conditions (i)-(iv) hold. Let $\tilde{u} \in \mathcal{M}_{-\pi, \pi}$ be a minimizer of $\tilde{J}$ on $\mathcal{M}_{-\pi, \pi}$, then

$$
\|\tilde{u}\|_{L^{\infty}(\mathbb{R})} \leq \frac{3}{2} \pi+\delta
$$

where

$$
\delta:=\frac{4 c_{1}^{2}}{c^{2}-c_{1}^{2}+c \sqrt{c^{2}-c_{1}^{2}}} .
$$

In particular, if the speed $c$ is large enough to ensure $\delta<\pi$, then $\|\tilde{u}\|_{L^{\infty}(\mathbb{R})} \leq \frac{5}{2} \pi$.

\section{Main result}

In order to prove the main result, we need the following version of the concentration-compactness principle obtained in [15, Lemma 4.1] (see $[16,17,19]$ for other versions of this principle).

Given $T>1$ and $\eta \in \mathbb{R}$, we define a truncated version of $\tilde{J}$ by

$$
\begin{aligned}
\tilde{J}_{T}(u, \eta) & :=\int_{0}^{1} \int_{\eta-T+\tau}^{\eta+T-1+\tau} \frac{c^{2}}{2}\left(u^{\prime}(s)\right)^{2} d s d \tau-\int_{\eta-T}^{\eta+T-1} V(A u(s)) d s \\
& -\int_{\eta-T}^{\eta+T-1} V(B u(s)) d s+\int_{\eta-T+\frac{1}{2}}^{\eta+T-\frac{1}{2}}\left[K(1+\cos (u(s)))+\frac{3}{2} F(u(s))\right] d s .
\end{aligned}
$$

Lemma 4.1 (Concentration-compactness). Assume conditions (i)-(iv) hold. Let $\left(u_{n}\right) \subset \mathcal{M}_{-\pi, \pi}$ be a minimizing sequence for $\tilde{J}$ on $\mathcal{M}_{-\pi, \pi}$, and let $c$ be large enough to ensure $\delta<\pi$ for $\delta$ defined in (13). Then there exists a subsequence, still denoted by $\left(u_{n}\right)$, such that one of the following holds: 
$\left(\mathrm{i}_{2}\right)$ (concentration) there is a sequence $\left(\eta_{n}\right) \subset \mathbb{R}$ such that for all small enough $\varepsilon>0$ there exists $T>0$ such that

$$
\left|\tilde{J}\left(u_{n}\right)-\tilde{J}_{T}\left(u_{n}, \eta_{n}\right)\right|<\varepsilon
$$

for every $n \in \mathbb{N}$;

(ii ${ }_{2}$ ) (vanishing) for all $T>0$,

$$
\lim _{n \rightarrow \infty} \sup _{\eta \in \mathbb{R}} \tilde{J}_{T}\left(u_{n}, \eta\right)=0
$$

(iii 2$)$ (dichotomy) there exists $\varepsilon_{1}>0$ such that for every $0<\varepsilon<\varepsilon_{1}$ there are $\left(f_{n}\right),\left(g_{n}\right) \subset E$ such that

$$
\begin{gathered}
\left|u_{n}-\left(f_{n}+g_{n}-\pi\right)\right| \leq \varepsilon, \quad \mid \tilde{J}\left(u_{n}\right)-\left(\tilde{J}\left(f_{n}\right)+\tilde{J}\left(g_{n}\right) \mid \leq \varepsilon,\right. \\
\lim _{n \rightarrow \infty} \operatorname{dist}\left(\operatorname{supp}\left(f_{n}^{\prime}\right), \operatorname{supp}\left(g_{n}^{\prime}\right)\right)=+\infty, \quad \lim _{n \rightarrow \infty} \tilde{J}\left(f_{n}\right)=\alpha, \lim _{n \rightarrow \infty} \tilde{J}\left(g_{n}\right)=\beta,
\end{gathered}
$$

for some $0<\alpha, \beta<\inf _{u \in \mathcal{M}_{-\pi, \pi}} \tilde{J}(u)$ ( $\pi$ is needed in the first inequality to ensure $J\left(f_{n}\right)<+\infty$ and $\left.J\left(g_{n}\right)<+\infty\right)$.

Lemma 4.2. Under the assumptions of Lemma 4.1, the functional $\tilde{J}$ has a minimizer on $\mathcal{M}_{-\pi, \pi}$.

Proof. By Lemma 3.4, the functional $\tilde{J}$ is bounded from below on $\mathcal{M}_{-\pi, \pi}$. Let $\left(u_{n}\right) \subset \mathcal{M}_{-\pi, \pi}$ be a minimizing sequence. Then, by Lemma 4.1, the subsequence exists, still denoted by $\left(u_{n}\right)$, which satisfies either of the following criteria: concentration, vanishing or dichotomy.

Vanishing is impossible (see the proof of Lemma 5.1 in [15]).

We will show that dichotomy is also impossible. Indeed, as $f_{n}, g_{n} \in E$ and $\tilde{J}\left(f_{n}\right), \tilde{J}\left(g_{n}\right)<+\infty$, the analogous statement of Lemma 3.1 (with $J$ replaced by $\tilde{J})$ shows that $f_{n}( \pm \infty), g_{n}( \pm \infty) \in\{ \pm \pi\}$. Since $f_{n}+g_{n}-\pi \in \mathcal{M}_{-\pi, \pi}$, then only $f_{n}(-\infty)=f_{n}(+\infty)$ or only $g_{n}(-\infty)=g_{n}(+\infty)$. In the first case, we set $\tilde{u}_{n}:=$ $g_{n}$ and in the second case, $\tilde{u}_{n}:=f_{n}$. Then $\left(\tilde{u}_{n}\right) \subset \mathcal{M}_{-\pi, \pi}$ and, by (iii 2$)$, possibly after passing to a subsequence, we have

$$
\lim _{n \rightarrow \infty} \tilde{J}\left(\tilde{u}_{n}\right)<\inf _{u \in \mathcal{M}_{-\pi, \pi}} \tilde{J}(u)=\lim _{n \rightarrow \infty} \tilde{J}\left(u_{n}\right) .
$$

We obtained a contradiction to the assumption that $\left(u_{n}\right) \subset \mathcal{M}_{-\pi, \pi}$ is a minimizing sequence of $\tilde{J}$.

Thus $\left(\mathrm{i}_{2}\right)$ holds. Hence, given $\varepsilon>0$, there exists a sequence $\left(\eta_{n}\right) \subset \mathbb{R}$ and $T_{0}>0$ such that

$$
\left|\tilde{J}\left(u_{n}\right)-\tilde{J}_{T_{0}}\left(u_{n}, \eta_{n}\right)\right|<\varepsilon .
$$

Let $w_{n}(s)=u_{n}\left(\eta_{n}+s\right)$. The sequence $\left(w_{n}\right)$ is bounded in $E$. Indeed, by (9),

$$
\left\|w_{n}^{\prime}\right\|_{L^{2}(\mathbb{R})}=\left\|u_{n}^{\prime}\right\|_{L^{2}(\mathbb{R})} \leq \frac{2}{c^{2}-c_{1}^{2}} J\left(u_{n}\right),
$$


and by Lemma 3.5,

$$
\left|w_{n}(0)\right| \leq \frac{3}{2} \pi+\delta
$$

Hence, $\left(w_{n}\right)$ contains a subsequence, still denoted by $\left(w_{n}\right)$, that converges weakly to some limit $u \in E$. The convergence is uniform on $\left[-T_{0}, T_{0}\right]$, and

$$
\left\|u^{\prime}\right\|_{L^{2}\left(-T_{0}, T_{0}\right)} \leq \lim _{n \rightarrow \infty} \inf \left\|w_{n}^{\prime}\right\|_{L^{2}\left(-T_{0}, T_{0}\right)} .
$$

Since the functions $V(u), 1+\cos u$ and $F(u)$ belong to $C^{1}(\mathbb{R})$ and therefore are Lipschitz continuous for $|u| \leq \frac{3}{2} \pi+\delta$, there exists $n_{0} \in \mathbb{N}$ such that for all $n>$ $n_{0}$,

$$
\left|\left(\tilde{J}(u)-\frac{c^{2}}{2}\left\|u^{\prime}\right\|_{L^{2}(\mathbb{R})}\right)-\left(\tilde{J}_{T_{0}}\left(w_{n}\right)-\frac{c^{2}}{2}\left\|u^{\prime}\right\|_{L^{2}\left(-T_{0}, T_{0}\right)}\right)\right| \leq \varepsilon .
$$

In fact, this inequality holds for all $T>T_{0}$ instead of $T_{0}$. By Lemma 3.1, $u \in$ $\mathcal{M}_{-\pi, \pi}$. Furthermore, as $T \mapsto \tilde{J}_{T}\left(w_{n}, 0\right)$ is non-decreasing for every $n \in \mathbb{N}$, we obtain that $\tilde{J}_{T}\left(w_{n}, 0\right) \leq \tilde{J}\left(w_{n}\right)$. Then,

$$
\begin{aligned}
\tilde{J}(u)=\lim _{T \rightarrow \infty} \tilde{J}_{T}(u, 0) & \leq \lim _{T \rightarrow \infty} \lim _{n \rightarrow \infty} \inf \tilde{J}_{T}\left(w_{n}, 0\right) \\
& \leq \lim _{T \rightarrow \infty} \lim _{n \rightarrow \infty} \tilde{J}\left(w_{n}\right)=\lim _{n \rightarrow \infty} \tilde{J}\left(w_{n}\right)=\lim _{n \rightarrow \infty} \tilde{J}\left(u_{n}\right),
\end{aligned}
$$

and thus $u$ is a minimizer of the functional $\tilde{J}$ on $\mathcal{M}_{-\pi, \pi}$.

The following theorem is the main result of the paper.

Theorem 4.3. Assume conditions (i)-(iv) hold. Suppose that $c$ is large enough to ensure $\delta<\pi$ for $\delta$ defined by (13). Then equation (2) has a solution $u$ that satisfies boundary conditions (3).

Proof. By Lemma 3.1, the modified functional $\tilde{J}$ has a minimizer $u_{*} \in \mathcal{M}_{-\pi, \pi}$. We have to show that $u_{*}$ is a solution of equation (2) with boundary conditions (3). We define the functional $\tilde{\Psi}$ similarly to $\Psi$ but in terms of $\tilde{J}$. Then the function $v_{*}=u_{*}-v_{0}$ minimizes $\tilde{\Psi}$ on $H^{1}(\mathbb{R})$. Since the embedding $H^{1}(\mathbb{R}) \subset$ $L^{\infty}(\mathbb{R})$ is continuous, we have that

$$
\left\|v_{0}+v\right\|_{L^{\infty}(\mathbb{R})}<\frac{5}{2} \pi
$$

for all $v$ in the neighborhood $\Delta \subset H^{1}(\mathbb{R})$ of $v_{*}$. Then, by Remark 3.2, for all $v \in \Delta$,

$$
\Psi(v)=J\left(v_{0}+v\right)=\tilde{J}\left(v_{0}+v\right)=\tilde{\Psi}(v),
$$

and $v_{*}$ minimizes $\Psi$ as well as $\tilde{\Psi}$ in $\Delta$. In particular, $v_{*}$ is a local minimizer of the functional $\Psi$ on $H^{1}(\mathbb{R})$, i.e., $v_{*}$ is a critical point of $\Psi$. Hence, by Lemma 3.1 (iv 1 ), $u_{*}=v_{0}+v_{*}$ is the solution of equation (2) that satisfies boundary conditions (3). 


\section{References}

[1] S.M. Bak, Traveling waves in chains of oscillators, Mat. Stud. 26 (2006), 140-153 (Ukrainian).

[2] S.M. Bak, Periodic traveling waves in chains of oscillators, Commun. Math. Anal. 3 (2007), 19-26.

[3] S.M. Bak, Existence of periodic traveling waves in a system of nonlinear oscillators on a two-dimensional lattice, Mat. Stud. 35 (2011), 60-65 (Ukrainian).

[4] S.M. Bak, Existence of periodic traveling waves in the Fermi-Pasta-Ulam system on a two-dimensional lattice, Mat. Stud. 37 (2012), 76-88 (Ukrainian).

[5] S.M. Bak, Periodic traveling waves in the discrete sine-Gordon equation on $2 \mathrm{D}$ lattice, Mat. Komp. Model. Ser.: Fiz.-Mat. Nauky 9 (2013), 5-10 (Ukrainian).

[6] S.M. Bak, Existence of the subsonic periodic traveling waves in the system of nonlinearly coupled nonlinear oscillators on 2D-lattice, Mat. Komp. Model. Ser.: Fiz.-Mat. Nauky 10 (2014), 17-23 (Ukrainian).

[7] S.M. Bak, Existence of the supersonic periodic traveling waves in the system of nonlinearly coupled nonlinear oscillators on 2D-lattice, Mat. Komp. Model. Ser.: Fiz.-Mat. Nauky 12 (2015), 5-12 (Ukrainian).

[8] S.M. Bak, Existence of heteroclinic traveling waves in a system of oscillators on a two-dimensional lattice, Mat. Metodi Fiz.-Mekh. Polya 57 (2014), 45-52 (Ukrainian); Engl. transl.: J. Math. Sci. (N.Y.) 217 (2016), 187-197.

[9] S.N. Bak, Existence of solitary traveling waves for a system of nonlinear coupled oscillators on a two-dimensional lattice, Ukraïn. Mat. Zh. 69 (2017), 435-444 (Ukrainian); Engl. transl.: Ukrainian Math. J. 69 (2017), 509-520.

[10] S.N. Bak and A.A. Pankov, Traveling waves in systems of oscillators on twodimensional lattices, Ukr. Mat. Visn. 7 (2010), 154-175 (Ukrainian); Engl. transl.: J. Math. Sci. (N.Y.) 174 (2011), 437-452.

[11] O.M. Braun and Y.S. Kivshar, The Frenkel-Kontorova Model. Concepts, Methods, and Applications. Texts and Monographs in Physics, Springer-Verlag, Berlin, 2004.

[12] M. Fečkan and V. Rothos, Travelling waves in Hamiltonian systems on 2D lattices with nearest neighbour interactions, Nonlinearity 20 (2007), 319-341.

[13] G. Friesecke and K. Matthies, Geometric solitary waves in a 2D math-spring lattice, Discrete Contin. Dyn. Syst. Ser. B 3 (2003), 105-114.

[14] G. Ioos and K. Kirchgässner, Travelling waves in a chain of coupled nonlinear oscillators, Comm. Math. Phys. 211 (2000), 439-464.

[15] C.-F. Kreiner and J. Zimmer, Heteroclinic travelling waves for the lattice sineGordon equation with linear pair interaction, Discrete Contin. Dyn. Syst. 25 (2009), 915-931.

[16] C.-F. Kreiner and J. Zimmer, Travelling wave solutions for the discrete sine-Gordon equation with nonlinear pair interaction, Nonlinear Anal. 70 (2009), 3146-3158.

[17] P.-L. Lions, The concentration-compactness principle in the calculus of variations. The locally compact case, I, II, Ann. Inst. H. Poincaré Anal. Non Linéaire 1 (1984), 223-283. 
[18] P.D. Makita, Periodic and homoclinic travelling waves in infinite lattices, Nonlinear Anal. 74 (2011), 2071-2086.

[19] A. Pankov, Travelling Waves and Periodic Oscillations in Fermi-Pasta-Ulam Lattices. Imperial College Press, London, 2005.

Received June 22, 2017.

S. Bak,

Vinnytsia Mykhailo Kotsiubynskyi State Pedagogical University, 32 Ostrozkogo St., Vinnytsia, 21001, Ukraine,

E-mail: sergiy.bak@gmail.com

\title{
Існування гетероклінічних рухомих хвиль в дискретному рівнянні синус-Гордона на двовимірній гратці
}

\author{
С. Бак
}

Статтю присвячено дискретному рівнянню синус-Гордона, яке описує нескінченну систему нелінійно зв'язаних нелінійних осциляторів на двовимірній гратці із зовнішнім потенціалом $V(r)=K(1-\cos r)$. Основний результат стосується існування розв'язків у вигляді гетероклінічних рухомих хвиль. За допомогою методу критичних точок і принципу концентрованої компактності отримано достатні умови існування таких розв'язків.

Ключові слова: дискретне рівняння синус-Гордона, нелінійні осцилятори, двовимірна гратка, гетероклінічні рухомі хвилі, критичні точки, принцип концентрованої компактності. 